\title{
State Feedback Control for Stochastic Feedforward Nonlinear Systems
}

\author{
Liang Liu ${ }^{1}$ and Ming Gao ${ }^{2}$ \\ ${ }^{1}$ College of Engineering, Bohai University, Liaoning 121013, China \\ ${ }^{2}$ College of Mathematics and Physics, Bohai University, Liaoning 121013, China \\ Correspondence should be addressed to Liang Liu; smithll@163.com
}

Received 13 October 2013; Accepted 13 December 2013

Academic Editor: Xudong Zhao

Copyright (c) 2013 L. Liu and M. Gao. This is an open access article distributed under the Creative Commons Attribution License, which permits unrestricted use, distribution, and reproduction in any medium, provided the original work is properly cited.

\begin{abstract}
This paper considers the state feedback stabilization problem for a class of stochastic feedforward nonlinear systems. By using the homogeneous domination approach, a state feedback controller is constructed to render the closed-loop system globally asymptotically stable in probability. A simulation example is provided to show the effectiveness of the designed controller.
\end{abstract}

\section{Introduction}

Consider the following stochastic feedforward nonlinear systems described by

$$
\begin{aligned}
d x_{1} & =x_{2} d t+f_{1}\left(\bar{x}_{3}, u\right) d t+g_{1}^{T}\left(\bar{x}_{2}, u\right) d \omega, \\
& \vdots \\
d x_{n-2} & =x_{n-1} d t+f_{n-2}\left(\bar{x}_{n}, u\right) d t+g_{n-2}^{T}\left(\bar{x}_{n-1}, u\right) d \omega, \\
d x_{n-1} & =x_{n} d t+f_{n-1}(u) d t+g_{n-1}^{T}\left(\bar{x}_{n}, u\right) d \omega, \\
d x_{n} & =u d t,
\end{aligned}
$$

where $x=\left(x_{1}, \ldots, x_{n}\right)^{T} \in R^{n}$ and $u \in R$ are the system state and input, respectively. $\bar{x}_{i}=\left(x_{i}, \ldots, x_{n}\right), i=2, \ldots, n$. $\omega$ is an $m$-dimensional standard Wiener process defined on the complete probability space $\left(\Omega, \mathscr{F},\left\{\mathscr{F}_{t}\right\}_{t \geq 0}, P\right)$ with $\Omega$ being a sample space, $\mathscr{F}$ being a $\sigma$-field, $\left\{\mathscr{F}_{t}\right\}_{t \geq 0}$ being a filtration, and $P$ being a probability measure. $f_{i}: R^{n-i-1} \times R \rightarrow R$ and $g_{i}: R^{n-i} \times R \rightarrow R^{m}$ are assumed to be locally Lipschitz with $f_{i}(0,0)=0$ and $g_{i}(0,0)=0, i=1, \ldots, n-1$.

Since the stochastic stability theory was established, the stabilization problems for stochastic lower-triangular nonlinear systems have made a great number of achievements in recent years; see, for example, [1-16] and the other references.
Feedforward system is an another important class of nonlinear systems. From the theoretical viewpoint, they are not feedback linearizable and cannot be stabilized by the conventional backstepping method; to some extent, the control problem of these systems is more difficult than feedback systems. On the other hand, some simple physical models, for example, the cart-pendulum system in [17] and the ball-beam with a friction term in [18], can be described by equations with the feedforward form. In recent papers on feedforward systems, [19] studied delay-adaptive feedback for linear systems. The input delay compensation for forward complete and strict-feedforward nonlinear systems was solved by [20]. Reference [21] considered the adaptive stabilization problem for feedforward nonlinear systems with time delays by taking a nested saturation feedback. The global output feedback stabilization problem for system (1) without stochastic noise was addressed by [22]. Reference [23] investigated the state and output feedback control for a class of feedforward nonlinear time-delay systems. For high-order nonlinear feedforward systems, [24] considered global stabilization problem by using the generalized adding a power integrator method and a series of nested saturation functions, $[25,26]$ respectively dealt with the state feedback control for this kind of systems with time delay, but all these results are limited to deterministic systems. Due to the special form of this system, there are few results on stochastic feedforward systems at present. 
The purpose of this paper is to solve the state feedback stabilization problem of system (1) by using the homogeneous domination approach in [22]. The underlying idea of this approach is that the homogeneous controller is first developed without considering the drift and diffusion terms, and then a low gain is introduced to the state feedback controller to dominate the drift and diffusion terms. By adopting this method, a state feedback controller is explicitly constructed to render the closed-loop system globally asymptotically stable in probability.

The paper is organized as follows. Section 2 provides some preliminary results. The design and analysis of state feedback controller is given in Sections 3 and 4, following a simulation example in Section 5. Section 6 concludes this paper.

\section{Preliminary Results}

The following notations, definitions, and lemmas are to be used throughout the paper.

$R_{+}$denotes the set of all nonnegative real numbers and $R^{n}$ denotes the real $n$-dimensional space. For a given vector or matrix $X, X^{T}$ denotes its transpose, $\operatorname{Tr}\{X\}$ denotes its trace when $X$ is square, and $|X|$ is the Euclidean norm of a vector $X$. $\mathscr{C}^{i}$ denotes the set of all functions with continuous $i$ th partial derivatives. $\mathscr{K}$ denotes the set of all functions: $R_{+} \rightarrow R_{+}$, which are continuous, strictly increasing, and vanishing at zero; $\mathscr{K}_{\infty}$ denotes the set of all functions which are of class $\mathscr{K}$ and unbounded; $\mathscr{K} \mathscr{L}$ denotes the set of all functions $\beta(s, t)$ : $R_{+} \times R_{+} \rightarrow R_{+}$, which are of $\mathscr{K}$ for each fixed $t$ and decrease to zero as $t \rightarrow \infty$ for each fixed $s$.

Consider the following stochastic nonlinear system:

$$
d x=f(x) d t+g^{T}(x) d \omega, \quad x(0)=x_{0} \in R^{n},
$$

where $x \in R^{n}$ is the system state and $\omega$ is an $m$-dimensional standard Wiener process defined on the complete probability space $\left(\Omega, \mathscr{F},\left\{\mathscr{F}_{t}\right\}_{t \geq 0}, P\right)$. The Borel measurable functions $f: R^{n} \rightarrow R^{n}$ and $g: R^{n} \rightarrow R^{m \times n}$ are locally Lipschitz with $f(0)=0$ and $g(0)=0$.

Definition 1 (see [1]). For any given $V(x) \in \mathscr{C}^{2}$ associated with stochastic system (2), the differential operator $\mathscr{L}$ is defined as

$$
\mathscr{L} V=\frac{\partial V}{\partial x} f(x)+\frac{1}{2} \operatorname{Tr}\left\{g(x) \frac{\partial^{2} V}{\partial x^{2}} g^{T}(x)\right\} .
$$

Definition 2 (see [1]). For system (2), the equilibrium $x=0$ is globally asymptotically stable (GAS) in probability if for any $\epsilon>0$, there exists a class $\mathscr{K} \mathscr{L}$ function $\beta(\cdot, \cdot)$ such that $P\left\{|x(t)|<\beta\left(\left|x_{0}\right|, t\right)\right\} \geq 1-\epsilon$ for any $t \geq 0$ and $x_{0} \in R^{n} \backslash\{0\}$.

Definition 3 (see [22]). For fixed coordinates $\left(x_{1}, \ldots, x_{n}\right) \in$ $R^{n}$ and real numbers $r_{i}>0, i=1, \ldots, n$.

(i) The dilation $\Delta_{\varepsilon}(x)$ is defined by $\Delta_{\varepsilon}(x)=\left(\varepsilon^{r_{1}} x_{1}, \ldots\right.$, $\left.\varepsilon^{r_{n}} x_{n}\right)$ for any $\varepsilon>0$ and $r_{1}, \ldots, r_{n}$ are called the weights of the coordinates. For simplicity, we define dilation weight as $\Delta=\left(r_{1}, \ldots, r_{n}\right)$. (ii) A function $V \in \mathscr{C}\left(R^{n}, R\right)$ is said to be homogeneous of degree $\tau$ if there is a real number $\tau \in R$ such that $V\left(\Delta_{\varepsilon}(x)\right)=\varepsilon^{\tau} V\left(x_{1}, \ldots, x_{n}\right)$ for any $x \in R^{n} \backslash\{0\}, \varepsilon>0$.

(iii) A vector field $h \in \mathscr{C}\left(R^{n}, R^{n}\right)$ is said to be homogeneous of degree $\tau$ if there is a real number $\tau \in R$ such that $h_{i}\left(\Delta_{\varepsilon}(x)\right)=\varepsilon^{\tau+r_{i}} h_{i}(x)$ for any $x \in R^{n} \backslash\{0\}, \varepsilon>0$, $i=1, \ldots, n$.

(iv) A homogeneous $p$-norm is defined as $\|x\|_{\Delta, p}=$ $\left(\sum_{i=1}^{n}\left|x_{i}\right|^{p / r_{i}}\right)^{1 / p}$ for any $x \in R^{n}$, where $p \geq 1$ is a constant. For simplicity, in this paper, we choose $p=2$ and write $\|x\|_{\Delta}$ for $\|x\|_{\Delta, 2}$.

Lemma 4 (see [1]). Consider system (2) and suppose that there exist a $\mathscr{C}^{2}$ function $V(x)$, class $\mathscr{K}_{\infty}$ functions $\alpha_{1}$ and $\alpha_{2}$, and a class $\mathscr{K}$ function $\alpha_{3}$ such that

$$
\alpha_{1}(|x|) \leq V(x) \leq \alpha_{2}(|x|), \quad \mathscr{L} V \leq-\alpha_{3}(|x|) .
$$

Then there exists an almost surely unique solution on $[0, \infty)$, the equilibrium $x=0$ is GAS in probability, and $P\left\{\lim _{t \rightarrow \infty}|x(t)|=0\right\}=1$.

Lemma 5 (see [22]). Given a dilation weight $\Delta=\left(r_{1}, \ldots, r_{n}\right)$, suppose that $V_{1}(x)$ and $V_{2}(x)$ are homogeneous functions of degrees $\tau_{1}$ and $\tau_{2}$, respectively. Then $V_{1}(x) V_{2}(x)$ is also homogeneous with respect to the same dilation weight $\Delta$. Moreover, the homogeneous degree of $V_{1} \cdot V_{2}$ is $\tau_{1}+\tau_{2}$.

Lemma 6 (see [22]). Suppose that $V: R^{n} \rightarrow R$ is a homogeneous function of degree $\tau$ with respect to the dilation weight $\Delta$; then

(i) $\partial V / \partial x_{i}$ is homogeneous of degree $\tau-r_{i}$ with $r_{i}$ being the homogeneous weight of $x_{i}$;

(ii) there is a constant $c$ such that $V(x) \leq c\|x\|_{\Delta}^{\tau}$. Moreover, if $V(x)$ is positive definite, then $V(x) \geq \underline{c}\|x\|_{\Delta}^{\tau}$, where $\underline{c}$ is a positive constant.

Lemma 7 (see [4]). Let $c$ and $d$ be positive constants. For any positive number $\bar{\gamma}$, then $|x|^{c}|y|^{d} \leq(c /(c+d)) \bar{\gamma}|x|^{c+d}+(d /(c+$ d)) $\bar{\gamma}^{(-c / d)}|y|^{c+d}$

\section{Design of State Feedback Controller}

3.1. Assumption. For system (1), we need the following Assumption.

Assumption 8. For $i=1, \ldots, n-1$, there exist positive constants $a_{1}$ and $a_{2}$ such that

$$
\begin{aligned}
& \left|f_{i}\left(\bar{x}_{i+2}, u\right)\right| \leq a_{1}\left(\left|x_{i+2}\right|+\cdots+\left|x_{n}\right|+|u|\right), \\
& \left|g_{i}\left(\bar{x}_{i+1}, u\right)\right| \leq a_{2}\left(\left|x_{i+1}\right|+\cdots+\left|x_{n}\right|+|u|\right),
\end{aligned}
$$

where $x_{n+1}=0$.

Remark 9. Obviously, system (1) satisfying Assumption 8 is a stochastic feedforward nonlinear system. As discussed in the deterministic feedforward references such as [21-26], that 
is, $g_{i}(\cdot) \equiv 0$ for $i=1, \ldots, n-1$, and stochastic feedforward reference [27], Assumption 8 is a general and frequently used condition.

Due to the special form of stochastic feedforward system, almost all the existing methods fail to be applicable to solve the stabilization problem of system (1). Based on this reason, the objective of this paper is to design a state feedback controller for system (1) such that the equilibrium of the closedloop system is globally asymptotically stable in probability.

To achieve this objective, we first introduce the following coordinate transformation:

$$
\eta_{i}=\frac{x_{i}}{\kappa^{i-1}}, \quad v=\frac{u}{\kappa^{n}}, \quad i=1, \ldots, n,
$$

where $0<\kappa<1$ is a designed constant. With the help of (6), (1) can be rewritten as

$$
\begin{aligned}
d \eta_{1}= & \kappa \eta_{2} d t+\bar{f}_{1}\left(\bar{\eta}_{3}, v\right) d t+\bar{g}_{1}^{T}\left(\bar{\eta}_{2}, v\right) d \omega, \\
& \vdots \\
d \eta_{n-2}= & \kappa \eta_{n-1} d t+\bar{f}_{n-2}\left(\bar{\eta}_{n}, v\right) d t+\bar{g}_{n-2}^{T}\left(\bar{\eta}_{n-1}, v\right) d \omega, \\
d \eta_{n-1} & =\kappa \eta_{n} d t+\bar{f}_{n-1}(v) d t+\bar{g}_{n-1}^{T}\left(\bar{\eta}_{n}, v\right) d \omega, \\
d \eta_{n} & =\kappa v d t,
\end{aligned}
$$

where $\bar{f}_{i}=f_{i} / \kappa^{i-1}$ and $\bar{g}_{i}=g_{i} / \kappa^{i-1}, i=1, \ldots, n-1$.

3.2. State Feedback Control of Nominal Nonlinear System. We construct a state feedback controller for the following nominal nonlinear system of (7):

$$
\begin{gathered}
d \eta_{i}=\kappa \eta_{i+1} d t, \quad i=1, \ldots, n-1, \\
d \eta_{n}=\kappa v d t .
\end{gathered}
$$

Step 1. Introducing $\xi_{1}=\eta_{1}$ and choosing $V_{1}\left(\eta_{1}\right)=(1 / 4) \xi_{1}^{4}$, by (3) and (8), it can be verified that $\mathscr{L} V_{1}=\kappa \xi_{1}^{3} \eta_{2}$. The first virtual controller

$$
\eta_{2}^{*}=-c_{11} \xi_{1}=:-\alpha_{1} \xi_{1}, \quad c_{11}>0
$$

leads to $\mathscr{L} V_{1} \leq-\kappa c_{11} \xi_{1}^{4}+\kappa \xi_{1}^{3}\left(\eta_{2}-\eta_{2}^{*}\right)$.

Step $i(i=2, \ldots, n)$. In this step, one can obtain the similar property for the $i$ th subsystem, which is presented by the following lemma.

Lemma 10. Suppose that at Step $i-1$ there are a set of virtual controllers $\eta_{1}^{*}, \ldots, \eta_{i}^{*}$ defined by

$$
\begin{gathered}
\eta_{1}^{*}=0, \quad \xi_{1}=\eta_{1}-\eta_{1}^{*}=\eta_{1}, \\
\eta_{k}^{*}=-\alpha_{k-1} \xi_{k-1}, \quad \xi_{k}=\eta_{k}-\eta_{k}^{*}, \quad k=2, \ldots, i,
\end{gathered}
$$

such that the $(i-1)$ th Lyapunov function $V_{i-1}\left(\bar{\eta}_{i-1}\right)=$ $(1 / 4) \sum_{j=1}^{i-1} \xi_{j}^{4}$ satisfies

$$
\mathscr{L} V_{i-1} \leq-\kappa \sum_{j=1}^{i-1} c_{i-1, j} \xi_{j}^{4}+\kappa \xi_{i-1}^{3}\left(\eta_{i}-\eta_{i}^{*}\right),
$$

where $\alpha_{j}, c_{i-1, j}, j=1, \ldots, i-1$, are positive constants. Then there exists a virtual control law $\eta_{i+1}^{*}=-\alpha_{i} \xi_{i}$ such that

$$
\mathscr{L} V_{i} \leq-\kappa \sum_{j=1}^{i} c_{i j} \xi_{j}^{4}+\kappa \xi_{i}^{3}\left(\eta_{i+1}-\eta_{i+1}^{*}\right),
$$

where $V_{i}\left(\bar{\eta}_{i}\right)=(1 / 4) \sum_{j=1}^{i} \xi_{j}^{4}$.

Proof. From (3), (8), (10), and (11), it follows that

$$
\begin{aligned}
\mathscr{L} V_{i} \leq & -\kappa \sum_{j=1}^{i-1} c_{i-1, j} \xi_{j}^{4}+\kappa \xi_{i}^{3} \eta_{i+1} \\
& +\kappa \xi_{i-1}^{3} \xi_{i}-\kappa \xi_{i}^{3} \sum_{k=1}^{i-1} \frac{\partial \eta_{i}^{*}}{\partial \eta_{k}} \eta_{k+1} .
\end{aligned}
$$

We concentrate on the last two terms on the right-hand side of (13).

Using (10) and Lemma 7, one obtains

$$
\begin{gathered}
\xi_{i-1}^{3} \xi_{i} \leq l_{i, i-1,1} \xi_{i-1}^{4}+\rho_{i 1} \xi_{i}^{4}, \\
-\xi_{i}^{3} \sum_{k=1}^{i-1} \frac{\partial \eta_{i}^{*}}{\partial \eta_{k}} \eta_{k+1} \\
\leq\left|\xi_{i}\right|^{3}\left|\sum_{k=1}^{i-1} \alpha_{i-1} \cdots \alpha_{k}\left(\xi_{k+1}-\alpha_{k} \xi_{k}\right)\right| \\
\leq\left|\xi_{i}\right|^{3}\left(\sum _ { k = 1 } ^ { i - 1 } \left(\alpha_{i-1} \cdots \alpha_{k-1}\right.\right. \\
\leq \sum_{k=1}^{i-1} l_{i k 2} \xi_{k}^{4}+\rho_{i 2} \xi_{i}^{4},
\end{gathered}
$$

where $l_{i, i-1,1}, l_{i k 2}(k=1, \ldots, i-1), \rho_{i 1}$, and $\rho_{i 2}$ are positive constants, $\alpha_{0}=0$.

Choosing

$$
\begin{gathered}
c_{i j}= \begin{cases}c_{i-1, j}-l_{i j 2}>0, & j=1, \ldots, i-2, \\
c_{i-1, i-1}-l_{i, i-1,1}-l_{i, i-1,2}>0, & j=i-1,\end{cases} \\
\eta_{i+1}^{*}=-\left(c_{i i}+\rho_{i 1}+\rho_{i 2}\right) \xi_{i}=:-\alpha_{i} \xi_{i}, \quad c_{i i}>0,
\end{gathered}
$$

and substituting (14)-(15) into (13), one gets the desired result.

At Step $n$, choosing $V_{n}\left(\bar{\eta}_{n}\right)=(1 / 4) \sum_{i=1}^{n} \xi_{i}^{4}$ and

$$
v=\eta_{n+1}^{*}=-\alpha_{n} \xi_{n}=-\left(\bar{\alpha}_{n} \eta_{n}+\bar{\alpha}_{n-1} \eta_{n-1}+\cdots+\bar{\alpha}_{1} \eta_{1}\right),
$$

by (3), (12), and (16), one gets

$$
\mathscr{L} V_{n} \leq-\kappa \sum_{i=1}^{n} c_{n i} \xi_{i}^{4}+\kappa \xi_{n}^{3}\left(v-\eta_{n+1}^{*}\right)=-\kappa \sum_{i=1}^{n} c_{n i} \xi_{i}^{4},
$$


where $\xi_{n}=\eta_{n}-\eta_{n}^{*}, \bar{\alpha}_{i}=\alpha_{n} \cdots \alpha_{i}, c_{n i}$, and $i=1, \ldots, n$, are positive constants. The system (7) and (16) can be written as

$$
d \eta=\kappa E(\eta) d t+F(\eta, v) d t+G^{T}(\eta, v) d \omega,
$$

where $\eta=\bar{\eta}_{n}=\left(\eta_{1}, \ldots, \eta_{n}\right)^{T}, E(\eta)=\left(\eta_{2}, \ldots, \eta_{n}, v\right)^{T}, F(\eta$, $v)=\left(\bar{f}_{1}, \ldots, \bar{f}_{n-1}, 0\right)^{T}$, and $G(\eta, v)=\left(\bar{g}_{1}, \ldots, \bar{g}_{n-1}, 0\right)$. Introducing the dilation weight $\Delta=(\underbrace{1,1, \ldots, 1}_{\text {for } \eta_{1}, \ldots, 1})$, by (10) and $V_{n}(\eta)=(1 / 4) \sum_{i=1}^{n} \xi_{i}^{4}$, one obtains

$$
\begin{aligned}
V_{n}\left(\Delta_{\varepsilon}(\eta)\right) & =\frac{1}{4} \sum_{i=1}^{n}\left(\varepsilon \eta_{i}+\alpha_{i-1} \varepsilon \eta_{i-1}+\cdots+\alpha_{i-1} \cdots \alpha_{1} \varepsilon \eta_{1}\right)^{4} \\
& =\varepsilon^{4} V_{n}(\eta),
\end{aligned}
$$

from which and Definition 3, we know that $V_{n}(\eta)$ is homogeneous of degree 4.

\section{Stability Analysis}

We state the main result in this paper.

Theorem 11. If Assumption 8 holds for the stochastic feedforward nonlinear system (1), under the state feedback controller $u=\kappa^{n} v$ and (16), then

(i) the closed-loop system has an almost surely unique solution on $[0, \infty)$;

(ii) the equilibrium at the origin of the closed-loop system is GAS in probability.

Proof. We prove Theorem 11 by three steps.

Step 1. Since $f_{i}$ and $g_{i}$ are assumed to be locally Lipschitz, so the system consisting of (7) and (16) satisfies the locally Lipschitz condition.

Step 2. By Lemma 6 and (17), there exists a positive constant $c_{01}$ such that

$$
\frac{\partial V_{n}}{\partial \eta} \kappa E(\eta) \leq-c_{01} \kappa\|\eta\|_{\Delta}^{4} .
$$

By Assumption 8, (6), (16), and $0<\kappa<1$, one has

$$
\begin{aligned}
\left|\bar{f}_{i}\left(\bar{\eta}_{i+2}, v\right)\right| & \leq \frac{a_{1}\left(\kappa^{i+1}\left|\eta_{i+2}\right|+\cdots+\kappa^{n-1}\left|\eta_{n}\right|+\kappa^{n}|v|\right)}{\kappa^{i-1}} \\
& \leq a_{1} \kappa^{2}\left(\left|\eta_{i+2}\right|+\cdots+\left|\eta_{n}\right|+|v|\right) \\
& \leq \lambda_{1} \kappa^{2}\|\eta\|_{\Delta},
\end{aligned}
$$

where $\lambda_{1}$ is a positive constant. According to Lemmas 5-6 and (21), one obtains

$$
\frac{\partial V_{n}}{\partial \eta} F(\eta, v)=\sum_{i=1}^{n-1} \frac{\partial V_{n}}{\partial \eta_{i}} \bar{f}_{i}\left(\bar{\eta}_{i+2}, v\right) \leq c_{02} \kappa^{2}\|\eta\|_{\Delta}^{4},
$$

where $c_{02}$ is a positive constant. Similar to (21), there is a positive constant $\lambda_{2}$ such that

$$
\left|\bar{g}_{i}\left(\bar{\eta}_{i+1}, v\right)\right| \leq \lambda_{2} \kappa\|\eta\|_{\Delta},
$$

from which and Lemmas 5-6, one leads to

$$
\begin{aligned}
& \frac{1}{2} \operatorname{Tr}\left\{G(\eta, v) \frac{\partial^{2} V_{n}}{\partial \eta^{2}} G^{T}(\eta, v)\right\} \\
& \leq \frac{1}{2} m \sqrt{m} \sum_{i, j=1}^{n-1}\left|\frac{\partial^{2} V_{n}}{\partial \eta_{i} \partial \eta_{j}}\right|\left|\bar{g}_{i}\left(\bar{\eta}_{i+1}, v\right)\right|\left|\bar{g}_{j}\left(\bar{\eta}_{j+1}, v\right)\right| \\
& \leq c_{03} \kappa^{2}\|\eta\|_{\Delta}^{4},
\end{aligned}
$$

where $c_{03}$ is a positive constant. By (3), (18), (20), (22), and (24), one has

$$
\begin{aligned}
\mathscr{L} V_{n}= & \frac{\partial V_{n}}{\partial \eta} \kappa E(\eta)+\frac{\partial V_{n}}{\partial \eta} F(\eta, v) \\
& +\frac{1}{2} \operatorname{Tr}\left\{G(\eta, v) \frac{\partial^{2} V_{n}}{\partial \eta^{2}} G^{T}(\eta, v)\right\} \\
\leq & -c_{01} \kappa\|\eta\|_{\Delta}^{4}+\left(c_{02}+c_{03}\right) \kappa^{2}\|\eta\|_{\Delta}^{4} \\
= & -\kappa\left(c_{01}-\left(c_{02}+c_{03}\right) \kappa\right)\|\eta\|_{\Delta}^{4} .
\end{aligned}
$$

Since $c_{01}$ is a constant independent of $c_{02}$ and $c_{03}$, by choosing

$$
0<\kappa<\kappa^{*}=: \min \left\{1, \frac{c_{01}}{c_{02}+c_{03}}\right\},
$$

(25) becomes $\mathscr{L} V_{n} \leq-c_{0}\|\eta\|_{\Delta}^{4}$, where $c_{0}$ is a positive constant.

By Steps 1-2 and Lemma 4, the system consisting of (7) and (16) has an almost surely unique solution on $[0, \infty), \eta=0$ is GAS in probability, and $P\left\{\lim _{t \rightarrow \infty}|\eta|=0\right\}=1$.

Step 3. Since (6) is an equivalent transformation, the closedloop system consisting of (1), $u=\kappa^{n} v$, and (16) has the same properties as the system (7) and (16). Theorem 11 holds.

Remark 12. This paper extends the homogeneous domination idea from deterministic systems to stochastic system (1) and explicitly constructs a state feedback controller. It should be emphasized that the rigorous proof of Theorem 11 is not an easy work.

\section{A Simulation Example}

Consider the following stochastic nonlinear system:

$$
\begin{gathered}
d x_{1}=x_{2} d t+\frac{1}{30} u d t+\frac{1}{5} \sin x_{2} d \omega, \\
d x_{2}=u d t .
\end{gathered}
$$

It is easy to verify that Assumption 8 is satisfied with $a_{1}=$ $1 / 30$ and $a_{2}=1 / 5$. 


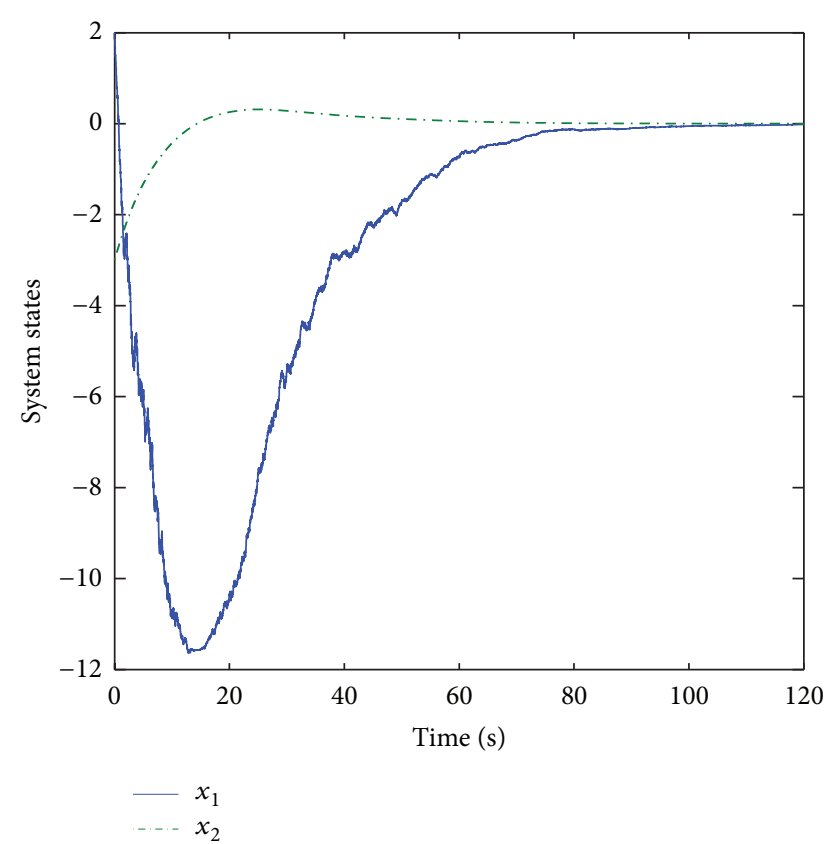

(a)

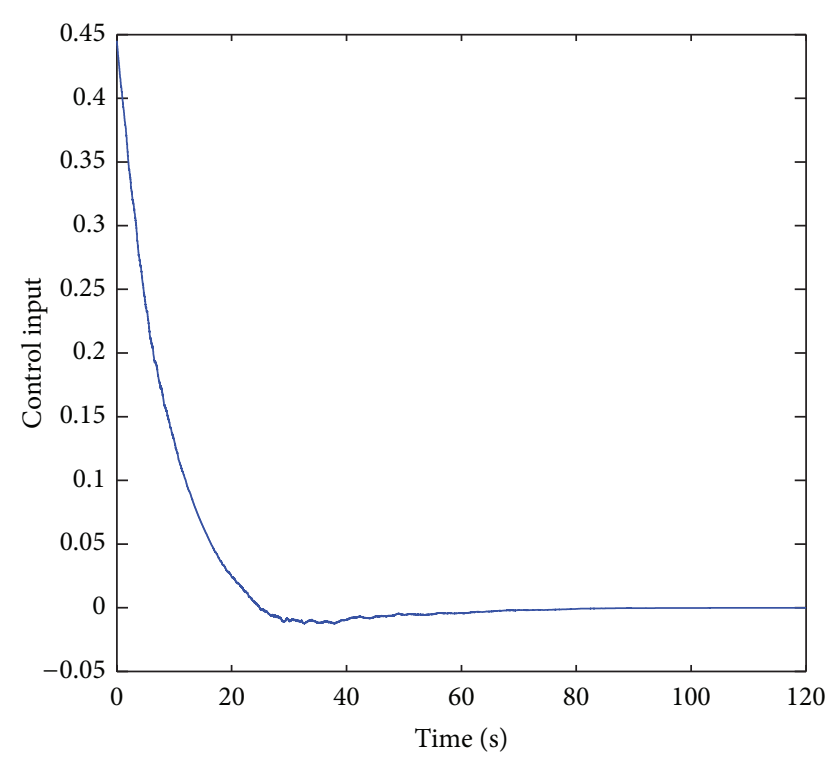

(b)

FIGURE 1: The responses of the closed-loop system (27) and (34).

Now, we give the controller design of system (27). Introducing the coordinate transformation

$$
\eta_{1}=x_{1}, \quad \eta_{2}=\frac{x_{2}}{\kappa}, \quad v=\frac{u}{\kappa^{2}},
$$

system (27) becomes

$$
\begin{gathered}
d \eta_{1}=\kappa \eta_{2} d t+\frac{\kappa^{2}}{30} v d t+\frac{1}{5} \sin \kappa \eta_{2} d \omega, \\
d \eta_{2}=\kappa v d t .
\end{gathered}
$$

Choosing $\xi_{1}=\eta_{1}$ and $V_{1}\left(\eta_{1}\right)=(1 / 4) \xi_{1}^{4}$, we obtain $\mathscr{L} V_{1} \leq$ $-2 \kappa \xi_{1}^{4}+\kappa \xi_{1}^{3}\left(\eta_{2}-\eta_{2}^{*}\right)$, where $\eta_{2}^{*}=-2 \eta_{1}=:-\alpha_{1} \xi_{1}$. By $\xi_{2}=$ $\eta_{2}-\eta_{2}^{*}$ and $V_{2}\left(\bar{\eta}_{2}\right)=V_{1}\left(\eta_{1}\right)+(1 / 4) \xi_{2}^{4}$, a direct calculation leads to

$$
\mathscr{L} V_{2} \leq-2 \kappa \xi_{1}^{4}+\kappa \xi_{1}^{3} \xi_{2}+\kappa \xi_{2}^{3} v+\kappa \alpha_{1} \xi_{2}^{3} \eta_{2}
$$

By Lemma 7, one has

$$
\begin{gathered}
\xi_{1}^{3} \xi_{2} \leq \frac{1}{2} \xi_{1}^{4}+\frac{27}{32} \xi_{2}^{4}, \\
\alpha_{1} \xi_{2}^{3} \eta_{2} \leq \frac{1}{2} \xi_{1}^{4}+(3 \sqrt[3]{2}+2) \xi_{2}^{4} .
\end{gathered}
$$

Choosing

$$
v=-\left(3+3 \sqrt[3]{2}+\frac{27}{32}\right) \xi_{2}=:-\alpha_{2} \xi_{2}
$$

and substituting (31) into (30), it leads to

$$
\mathscr{L} V_{2} \leq-\kappa\left(\xi_{1}^{4}+\xi_{2}^{4}\right) .
$$

By (28) and (32), one obtains the actual controller

$$
u=-\alpha_{2}\left(\kappa x_{2}+\alpha_{1} \kappa^{2} x_{1}\right)
$$

Defining $\|\eta\|_{\Delta}=\left(\eta_{1}^{2}+\eta_{2}^{2}\right)^{1 / 2}$, one gets $\mathscr{L} V_{2} \leq-\kappa\|\eta\|_{\Delta}^{4}$. From (22) and (24), it follows that

$$
\begin{gathered}
\frac{\partial V_{2}}{\partial \eta_{1}} \cdot \frac{\kappa^{2}}{30} v \leq 46.7133 \kappa^{2}\|\eta\|_{\Delta}^{4}, \\
\frac{1}{2} \frac{\partial^{2} V_{2}}{\partial \eta_{1}^{2}} \cdot \frac{1}{25} \sin ^{2} \kappa \eta_{2} \leq 2 \kappa^{2}\|\eta\|_{\Delta}^{4} .
\end{gathered}
$$

By (29), one has $\mathscr{L} V_{2} \leq-\kappa(1-48.7133 \kappa)\|\eta\|_{\Delta}^{4}$, from which one obtains $\kappa^{*}=0.0205$.

In simulation, we choose the initial values $x_{1}(0)=2$, $x_{2}(0)=-3$, and $\kappa=0.02$. Figure 1 demonstrates the effectiveness of the state feedback controller.

\section{A Concluding Remark}

In this paper, the homogeneous domination approach is introduced to solve the state feedback stabilization problem for the stochastic feedforward nonlinear system (1). There still exist some problems to be investigated. One is to consider the more general switched stochastic feedforward nonlinear systems by adopting average dwell time method in [28]. Another is to consider stochastic feedforward networked or fuzzy systems (similar to [29-33]).

\section{Acknowledgments}

The authors would like to express their sincere gratitude to the editor and reviewers for their helpful suggestions in improving the quality of this paper. This work was partially supported by the National Natural Science Foundation of China (nos. 61304002, 61304003, 61203123, and 61304054), the Fundamental Research Funds for the Central Universities of China (no. 11CX04044A), the Shandong Provincial Natural Science Foundation of China (no. ZR2012FQ019), and Doctoral Start-up Fund of Bohai University. 


\section{References}

[1] M. Krstic and H. Deng, Stabilization of Uncertain Nonlinear Systems, Springer, New York, NY, USA, 1998.

[2] Y. Li, "Exponential stability results of discrete-time stochastic neural networks with time-varying delays," Mathematical Problems in Engineering, Article ID 486257, 10 pages, 2013.

[3] L. Liu and N. Duan, "State-feedback stabilization for stochastic high-order nonlinear systems with a ratio of odd integers power," Nonlinear Analysis: Modelling and Control, vol. 15, no. 1, pp. 39-53, 2010.

[4] L. Liu and X. J. Xie, "Output-feedback stabilization for stochastic high-order nonlinear systems with time-varying delay," Automatica, vol. 47, no. 12, pp. 2772-2779, 2011.

[5] L. Liu and X. J. Xie, "State-feedback stabilization for stochastic high-order nonlinear systems with SISS inverse dynamics," Asian Journal of Control, vol. 14, no. 1, pp. 207-216, 2012.

[6] S. J. Liu, J. F. Zhang, and Z. P. Jiang, "Decentralized adaptive output-feedback stabilization for large-scale stochastic nonlinear systems," Automatica, vol. 43, no. 2, pp. 238-251, 2007.

[7] S. J. Liu, Z. P. Jiang, and J. F. Zhang, "Global output-feedback stabilization for a class of stochastic non-minimum-phase nonlinear systems," Automatica, vol. 44, no. 8, pp. 1944-1957, 2008.

[8] Y. G. Liu and J. F. Zhang, "Practical output-feedback risk-sensitive control for stochastic nonlinear systems with stable zerodynamics," SIAM Journal on Control and Optimization, vol. 45, no. 3, pp. 885-926, 2006.

[9] L. Wu, P. Shi, and H. Gao, "State estimation and slidingmode control of Markovian jump singular systems," IEEE Transactions on Automatic Control, vol. 55, no. 5, pp. 1213-1219, 2010.

[10] Z. J. Wu, X. J. Xie, and S. Y. Zhang, "Adaptive backstepping controller design using stochastic small-gain theorem," Automatica, vol. 43, no. 4, pp. 608-620, 2007.

[11] X. J. Xie and L. Liu, "Further results on output feedback stabilization for stochastic high-order nonlinear systems with timevarying delay," Automatica, vol. 48, no. 10, pp. 2577-2586, 2012.

[12] X. J. Xie and L. Liu, "A homogeneous domination approach to state feedback of stochastic high-order nonlinear systems with time-varying delay," IEEE Transactions on Automatic Control, vol. 58, no. 2, pp. 494-499, 2013.

[13] X. Yu and X. J. Xie, "Output feedback regulation of stochastic nonlinear systems with stochastic iISS inverse dynamics," IEEE Transactions on Automatic Control, vol. 55, no. 2, pp. 304-320, 2010.

[14] L. Zhang, E. K. Boukas, and J. Lam, "Analysis and synthesis of Markov jump linear systems with time-varying delays and partially known transition probabilities," IEEE Transactions on Automatic Control, vol. 53, no. 10, pp. 2458-2464, 2008.

[15] C. R. Zhao, X. J. Xie, and N. Duan, "Adaptive state-feedback stabilization for high-order stochastic nonlinear systems driven by noise of unknown covariance," Mathematical Problems in Engineering, Article ID 246579, 13 pages, 2012.

[16] X. Zhao, M. Ling, and Q. Zeng, "Delay-dependent robust control for uncertain stochastic systems with Markovian switching and multiple delays," Journal of Systems Engineering and Electronics, vol. 21, no. 2, pp. 287-295, 2010.

[17] F. Mazenc and S. Bowong, "Tracking trajectories of the cartpendulum system," Automatica, vol. 39, no. 4, pp. 677-684, 2003.

[18] R. Sepulchre, M. Janković, and P. V. Kokotović, Constructive Nonlinear Control, Springer, London, UK, 1997.
[19] N. Bekiaris-Liberis and M. Krstic, "Delay-adaptive feedback for linear feedforward systems," Systems \& Control Letters, vol. 59, no. 5, pp. 277-283, 2010.

[20] M. Krstic, "Input delay compensation for forward complete and strict-feedforward nonlinear systems," IEEE Transactions on Automatic Control, vol. 55, no. 2, pp. 287-303, 2010.

[21] X. Ye, "Adaptive stabilization of time-delay feedforward nonlinear systems," Automatica, vol. 47, no. 5, pp. 950-955, 2011.

[22] C. Qian and J. Li, "Global output feedback stabilization of upper-triangular nonlinear systems using a homogeneous domination approach," International Journal of Robust and Nonlinear Control, vol. 16, no. 9, pp. 441-463, 2006.

[23] X. Zhang, L. Baron, Q. Liu, and E. K. Boukas, "Design of stabilizing controllers with a dynamic gain for feedforward nonlinear time-delay systems," IEEE Transactions on Automatic Control, vol. 56, no. 3, pp. 692-697, 2011.

[24] S. Ding, C. Qian, S. Li, and Q. Li, "Global stabilization of a class of upper-triangular systems with unbounded or uncontrollable linearizations," International Journal of Robust and Nonlinear Control, vol. 21, no. 3, pp. 271-294, 2011.

[25] X. Zhang, E. K. Boukas, Y. Liu, and L. Baron, "Asymptotic stabilization of high-order feedforward systems with delays in the input," International Journal of Robust and Nonlinear Control, vol. 20, no. 12, pp. 1395-1406, 2010.

[26] X. Zhang, Q. Liu, L. Baron, and E. K. Boukas, "Feedback stabilization for high order feedforward nonlinear time-delay systems," Automatica, vol. 47, no. 5, pp. 962-967, 2011.

[27] L. Liu and X. J. Xie, "State feedback stabilization for stochastic feedforward nonlinear systems with time-varying delay," Automatica, vol. 49, no. 4, pp. 936-942, 2013.

[28] L. Zhang and H. Gao, "Asynchronously switched control of switched linear systems with average dwell time," Automatica, vol. 46, no. 5, pp. 953-958, 2010.

[29] H. Gao and T. Chen, " $H_{\infty}$ estimation for uncertain systems with limited communication capacity," IEEE Transactions on Automatic Control, vol. 52, no. 11, pp. 2070-2084, 2007.

[30] H. Gao, T. Chen, and J. Lam, "A new delay system approach to network-based control," Automatica, vol. 44, no. 1, pp. 39-52, 2008.

[31] H. Gao and T. Chen, "Network-based $H_{\infty}$ output tracking control," IEEE Transactions on Automatic Control, vol. 53, no. 3, pp. 655-667, 2008.

[32] H. Gao, X. Meng, and T. Chen, "Stabilization of networked control systems with a new delay characterization," IEEE Transactions on Automatic Control, vol. 53, no. 9, pp. 2142-2148, 2008.

[33] X. D. Zhao, L. X. Zhang, P. Shi, and H. R. Karimi, "Novel stability criteria for T-S fuzzy systems," IEEE Transactions on Fuzzy Systems, vol. 21, no. 6, pp. 1-11, 2013. 


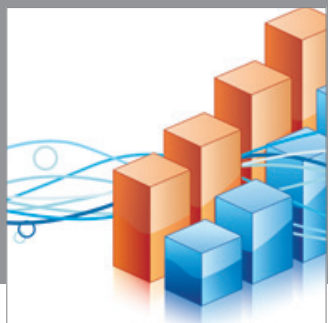

Advances in

Operations Research

mansans

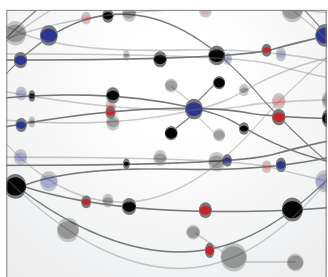

The Scientific World Journal
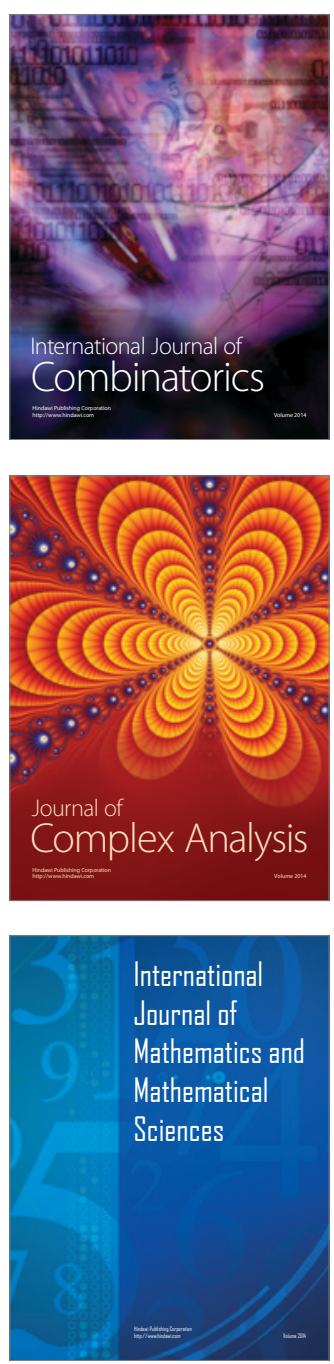
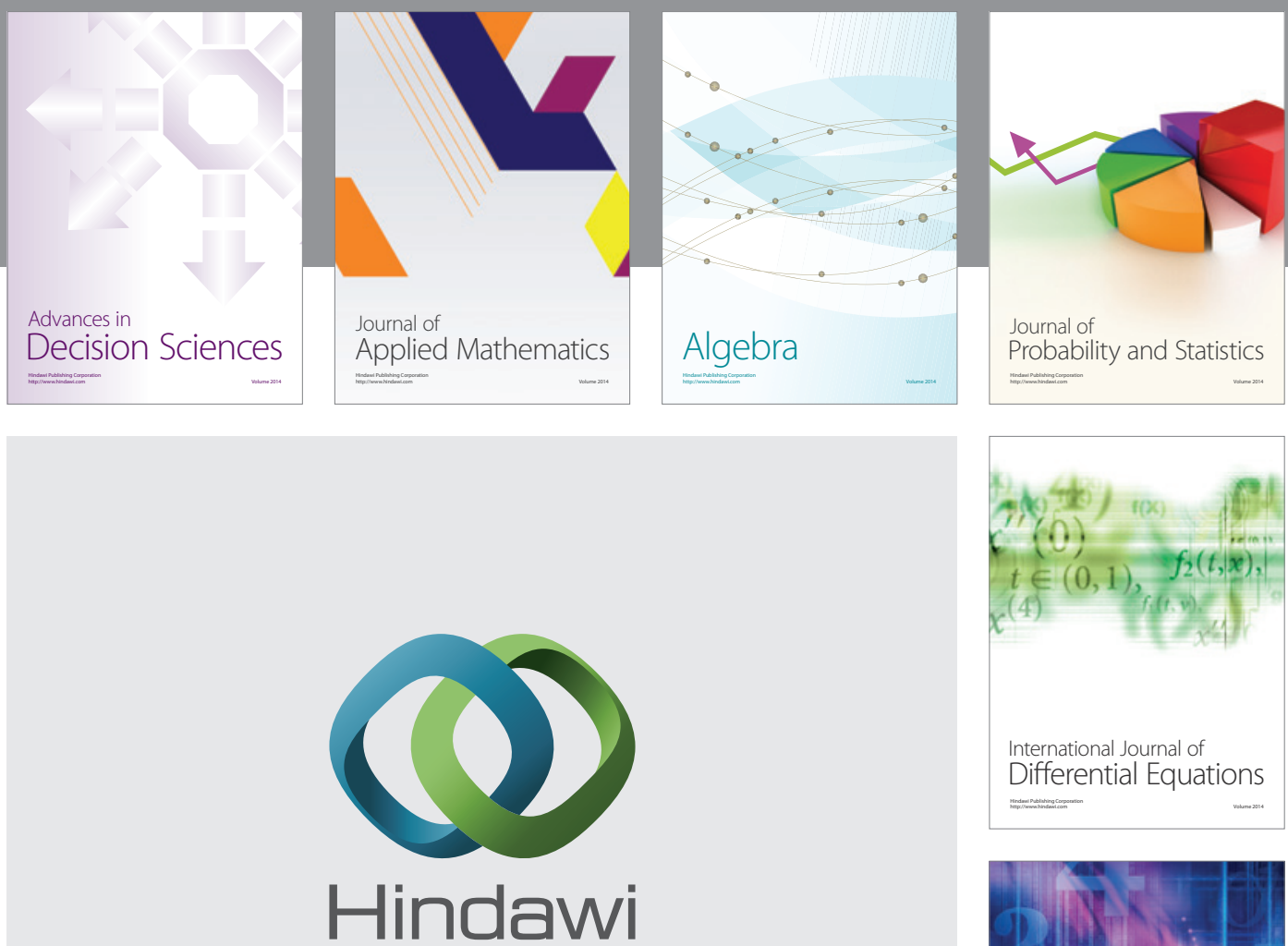

Submit your manuscripts at http://www.hindawi.com
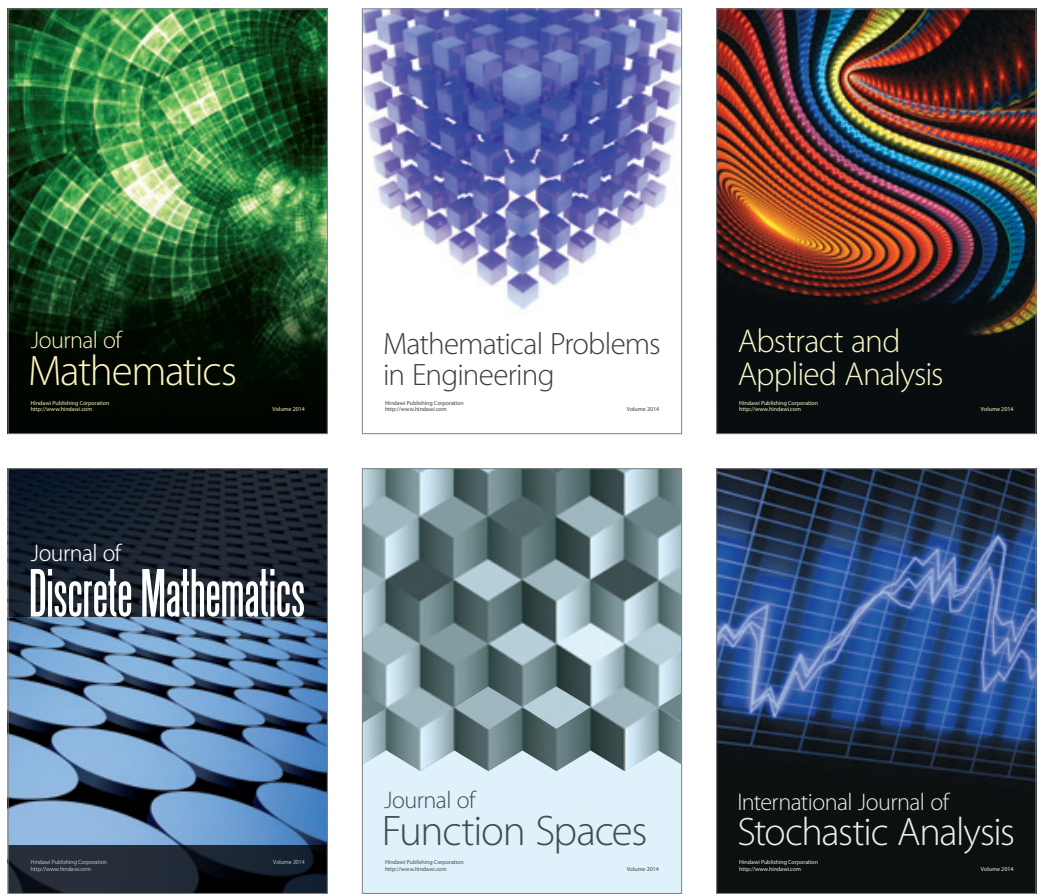

Journal of

Function Spaces

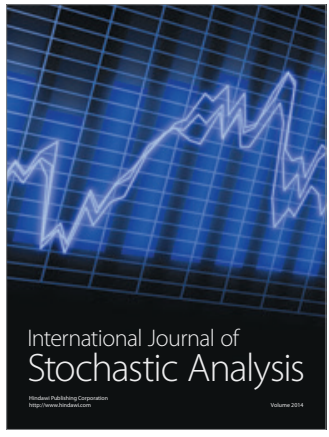

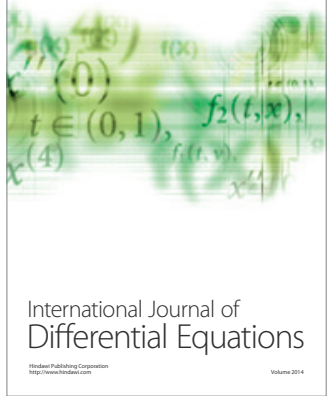
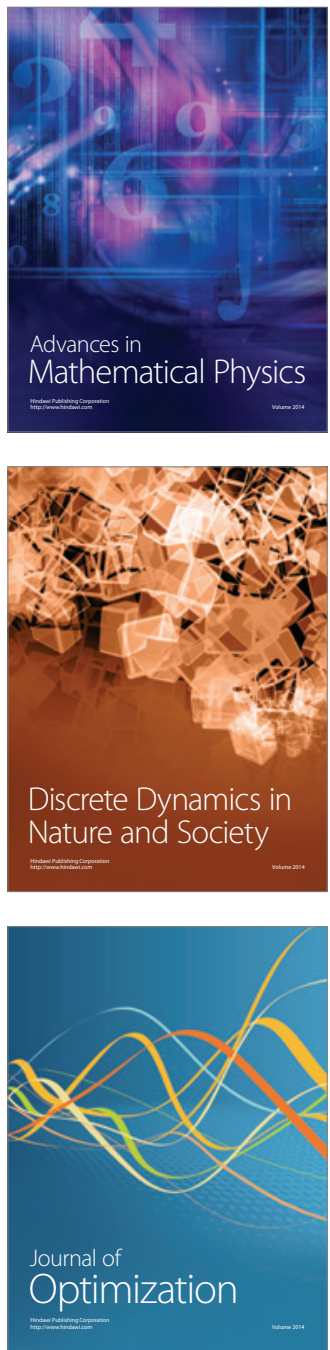